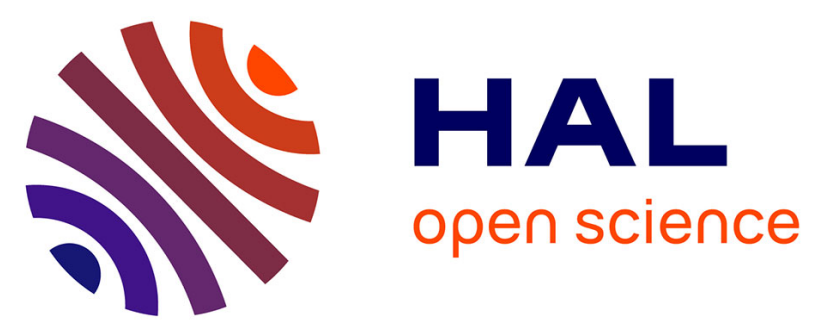

\title{
Socio-demographic factors associated with loss to follow-up of HIV infected women attending a private sector PMTCT program in Maharashtra, India
}

Mayuri Vijaykumar Panditrao, Shrinivas Satyanarayan Darak, Vinay Vaman

Kulkarni, Sanjeevani Vinay Kulkarni, Ritu Shridhar Parchure

\section{To cite this version:}

Mayuri Vijaykumar Panditrao, Shrinivas Satyanarayan Darak, Vinay Vaman Kulkarni, Sanjeevani Vinay Kulkarni, Ritu Shridhar Parchure. Socio-demographic factors associated with loss to follow-up of HIV infected women attending a private sector PMTCT program in Maharashtra, India. AIDS Care, 2011, 23 (05), pp.593-600. 10.1080/09540121.2010.516348 . hal-00665441

\section{HAL Id: hal-00665441 \\ https://hal.science/hal-00665441}

Submitted on 2 Feb 2012

HAL is a multi-disciplinary open access archive for the deposit and dissemination of scientific research documents, whether they are published or not. The documents may come from teaching and research institutions in France or abroad, or from public or private research centers.
L'archive ouverte pluridisciplinaire HAL, est destinée au dépôt et à la diffusion de documents scientifiques de niveau recherche, publiés ou non, émanant des établissements d'enseignement et de recherche français ou étrangers, des laboratoires publics ou privés. 

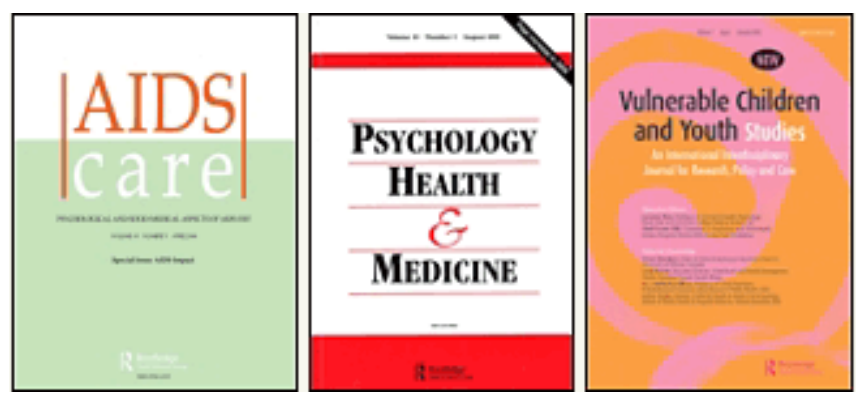

\section{Socio-demographic factors associated with loss to follow-up of HIV infected women attending a private sector PMTCT program in Maharashtra, India}

\begin{tabular}{|r|l|}
\hline Journal: & $\begin{array}{l}\text { AIDS Care - Psychology, Health \& Medicine - Vulnerable Children } \\
\text { and Youth Studies }\end{array}$ \\
\hline Manuscript ID: & AC-2010-04-0189.R1 \\
\hline Journal Selection: & AIDS Care \\
\hline Keywords: & PMTCT, India, HIV, loss to follow-up, health care utilization \\
\hline \multicolumn{2}{|c}{} \\
\hline
\end{tabular}

\section{SCHOLARONE"}

Manuscripts 
Socio-demographic factors associated with loss to follow-up of HIV infected women attending a private sector PMTCT program in Maharashtra, India

Abstract Word Count: 292

Paper Word Count: 2990

Key Words: PMTCT, India, HIV, loss to follow-up, health care utilization 


\section{Abstract}

Currently 40 percent of HIV infected women enrolled in national PMTCT program in India are lost to follow-up (LTF) before they can receive single dose Nevirapine. To date no study from India has examined the reasons for inadequate utilization of PMTCT services. This study sought to examine the socio-demographic factors associated with LTF of HIV infected women enrolled during 2002 to 2008 in a large-scale private sector PMTCT project in Maharashtra, India. Data on HIV infected women who were enrolled during pregnancy $(\mathrm{N}=734)$ and who reported live birth $(\mathrm{N}=770)$ were used to analyze factors associated with LTF before delivery and after delivery respectively. Univariate and multivariate analyses were conducted to estimate the associations between being LTF and socio-demographic factors using generalized linear models. Eighty (10.9\%) women were LTF before delivery and $151(19.6 \%)$ women were LTF after delivery. Women with less than graduate level education $(R R=6.32)$, from a poor family $(R R=1.61)$, who were registered after 20 weeks of pregnancy $(R R=2.02)$ and whose partners were HIV noninfected or with unknown HIV status $(\mathrm{RR}=2.69)$ were more likely to be LTF before delivery. Similarly, the significant factors for LTF after delivery were less than graduate level education $(R R=1.82)$, poor family $(R R=1.42)$ and registration after 20 weeks of pregnancy $(R R=1.75)$. This study highlights the need for innovative and effective counseling techniques for less educated women, economic empowerment of women, better strategies to increase up-take of partner's HIV testing and early registration of women in the program for preventing LTF in PMTCT programs. This need for innovative counseling techniques is even greater for PMTCT programs in the public health sector as the women accessing care in the public sector are likely to be less educated and economically more deprived. 


\section{Background}

Prevention of HIV transmission from a HIV infected pregnant woman to her child requires completion of a cascade of services beginning with HIV testing and counseling for the pregnant woman followed by antiretroviral treatment (ART) or prophylaxis for the HIV infected pregnant woman, safe obstetric interventions and counseling, and support for safer infant feeding options (World Health Organization [WHO], 2008). Adequate utilization of each service in this cascade contributes to determining the effectiveness of a program for prevention of mother to child transmission (PMTCT) of HIV.

Loss to follow-up (LTF) of women enrolled in a PMTCT program at different stages has been recognized as a major hurdle by PMTCT programs in resource poor settings. In one of the PMTCT projects in Malawi, the progressive LTF rates were 68 percent by delivery and 81 percent by the 6 month post-natal visit (Manzi et al., 2005). A PMTCT program from Côte d'Ivoire reported LTF rates of up to 84 percent before the women had started taking Zidovudine prophylaxis (Painter et al., 2004). Similarly a program in Zimbabwe reported 76 percent LTF rates before the mother and the baby could be given single dose Nevirapine (sdNVP) (Perez et al., 2004). While a study from South Africa reported that more than 70 percent of infants born to HIV infected mothers were LTF by 4 months of age (Sherman et al., 2004). In India more than 3.5 million pregnant women were tested for HIV in 2008 (National AIDS Control Organization [NACO], 2008). However, approximately 40 percent of the identified HIV infected women and their babies were apparently LTF before they could be given sdNVP (NACO, 2008). With high rates of LTF, efficacy of a PMTCT program is substantially reduced not only because the objective of reducing pediatric HIV transmissions is compromised but also because of the 
missed opportunity to link HIV infected women and their partners to further care and support activities.

There is dearth of literature on the risk factors for LTF of HIV infected women within PMTCT programs. Almost all of the studies in the PMTCT setting to date have been carried out in African countries. However factors found to be associated with LTF in the African PMTCT setting may be different from the Indian PMTCT setting due to substantial differences in the social, cultural, and economic context in which women's lives are embedded. Therefore, in order to increase the efficacy of a PMTCT program, there is a need to study the risk factors in the local and cultural contexts while identifying any common factors in the different geographic and cultural settings. Further the factors identified in previous studies have been mostly derived from qualitative data (Bwirire et al., 2008; Chinkonde et al., 2009). Previous qualitative studies in Africa have identified a few risk factors for LTF such as fear of stigma, discrimination, household conflict and even divorce on disclosure of HIV status; lack of support from partners; social and cultural taboos associated with artificial feeding; long waiting times at ANC; and unaffordable transport costs to the hospital (Bwirire et al., 2008; Chinkonde et al., 2009; Painter et al., 2004).

Although LTF in the PMTCT setting has been identified as a problem in India, no data on risk factors for LTF in PMTCT programs are available. Therefore understanding the risk factors for LTF of HIV infected women in the PMTCT programs in India will assist in devising strategies to achieve higher follow-up rates and subsequently improve health outcomes and survival in women and infants seeking care at such facilities. Hence the contributions of this paper are twofold: first, quantitative analysis of risk factors 
associated with LTF of HIV infected women in the PMTCT setting and second, focus on a geographic site where such a study has never been carried out previously. Quantitative analyses of the data are needed for better generalizability of the results. This study is also unique as it analyzes the data from one of the largest private sector PMTCT programs in the country. Therefore the findings of this study will be useful to the health care providers as well as to the program managers and policy makers currently implementing or planning to implement PMTCT programs in the private and public sectors.

\section{Methods}

\subsection{The PMTCT program}

A non-government organization (NGO) located in the city of Pune, Maharashtra, a state with HIV prevalence of 0.67 percent (NACO, 2007) started implementing a PMTCT program in the private health sector in September 2002. This program is one of the largest private sector PMTCT programs in India. By the end of 2008 the NGO collaborated with 43 hospitals in 9 districts of Maharashtra and provided comprehensive antenatal care (ANC) counseling and HIV testing services to 122,005 pregnant women and enrolled 950 HIV infected women in the PMTCT program. ANC and PMTCT counseling is provided by trained counselors located at these hospitals. HIV infected women are offered AZT based ARV prophylaxis for PMTCT as per the WHO recommendation for resource poor countries. Infant feeding counseling is provided to women and the option of breastfeeding as well as replacement feeding is given to women after thorough discussion on the availability, affordability, feasibility, accessibility and sustainability (AAFAS) of replacement feeding. HIV exposed infants are tested for HIV by DNA PCR test. 


\subsection{Data collection}

All data were collected by trained counselors located at the hospitals using a pre-designed semi-structured questionnaire. These data were collected primarily for programmatic purposes and not to study LTF in the PMTCT setting. The socio-demographic data were collected at the time of registration of pregnant women in the PMTCT project and the data on each follow-up visit were recorded on separate follow-up sheets. Case to case data validation was regularly conducted as a part of internal monitoring of the project and these data were entered in special software devised for monitoring the PMTCT project.

Detailed information was collected on socio-demographic factors, past obstetric and medical history of the woman, and socio-demographic information about partner. Information on delivery was noted on a separate delivery sheet. Detailed information on adherence to ART and infant feeding practices was collected during the follow-up visits.

\subsection{Case definition}

HIV infected women registered during pregnancy and who were not known to have terminated the pregnancy were included in the analysis. Women were considered as LTF before delivery if they did not come to the hospital for delivery by the expected date (cut off date was $31^{\text {st }}$ December 2008) and could not be contacted even after repeated attempts. Similarly women were considered LTF after delivery if they came during labor and delivered a live baby but did not come for regular post-natal visits till the baby's HIV status was diagnosed. As a routine procedure, consent for contacting women is sought at the time of registration into the program and women are contacted by letter, phone calls or home visits when they miss their scheduled visit with the doctor or the counselor. 


\subsection{Statistical Analyses}

We undertook a descriptive analysis of basic demographic characteristics of the HIV infected women enrolled in the project. We conducted statistical analyses to determine the risk factors for LTF before delivery and after delivery for pregnant women registered in the PMTCT program. Univariate analysis using generalized linear regression were conducted to estimate the associations between being LTF and demographic as well as other social factors. Statistically significant covariates (in the univariate analysis) were tested in multivariate models using generalized linear regression. Separate final models for analysis of LTF before delivery and after delivery were selected using backward stepwise elimination technique. All statistical analyses were performed with STATA for Windows (Version 9.0).

\section{Results}

Between 2002 and 2008, a total 950 HIV infected women were registered in the program (840 were enrolled during pregnancy and 110 were enrolled during labor). Of these women, $80(10.9 \%)$ women meeting the case definition of LTF before delivery and 151 (19.6\%) women meeting the case definition of LTF after delivery were identified. Table 1 shows results of univariate analysis (using regression techniques) of various potential factors associated with LTF before and after delivery. Presence of previous healthy children, woman's education level, family's economic status, partner's HIV status, and woman's referral status were found to be statistically associated with being LTF before delivery. In addition to these factors, place of residence, stage of pregnancy at the time of registration, mode of delivery, birth weight of baby, infant feeding option, and 
medication regimen given to the baby were found to be statistically associated with being LTF after delivery.

The results of multivariate analysis conducted to identify the risk factors associated with LTF before delivery are shown in Table 2.

A woman with less than graduate level education was 6.32 (95\% CI: 1.57-25.44) times more likely to be LTF than a woman with graduate level education after controlling for other covariates. A woman from a poor family was 1.61 (95\% CI: 1.07-2.42) times more likely to be LTF than a woman from a middle-class or rich family. A woman who was registered after 20 weeks of pregnancy was 2.02 (95\% CI: 1.34-3.05) times more likely to be LTF than a woman who was registered within the first 20 weeks of pregnancy. A woman whose partner's HIV status was seronegative or unknown was $2.69(95 \%$ CI: 1.71-4.24) times more likely to be LTF than a woman whose partner was HIV seropositive.

The results of multivariate analysis conducted to identify the risk factors associated with the woman's LTF after delivery are shown in Table 3.

A woman with less than graduate level education was 1.82 (95\% CI: 1.03-3.22) times more likely to be LTF than a woman with graduate level or higher than graduate level education after controlling for other covariates. A woman from a poor family was 1.42 (95\% CI: 1.05-1.91) times more likely to be LTF than a woman from a middle-class or rich family. A woman who was registered after 20 weeks of pregnancy was 1.75 (95\% CI: 1.12-2.73) times more likely to be LTF than a woman who was registered within the first 20 weeks of pregnancy. 


\section{Discussion}

Our analyses of the risk factors for LTF in HIV infected pregnant women before delivery and after delivery are discussed below in detail.

\section{Woman's education level}

Women with graduate level education were less likely to be LTF compared to women with less than graduate level education. Previous research in India on nationally representative sample has identified woman's education as a significant factor influencing utilization of maternal and child health care services (Govindasamy \& Ramesh,1997). Our analysis suggests that woman's education is an important determinant for continued utilization of PMTCT services among HIV infected women.

The relationship of maternal education and health care utilization is not straightforward and several pathways in which education may influence maternal health care utilization have been suggested. Educated women are better able to break away from the traditions to utilize modern means of safeguarding their own health, (Caldwell 1979; Caldwell \& Caldwell 1988; Cleland 1990) they are able to make independent decisions, (Caldwell 1979; Caldwell 1986) and utilize what is available in the community for their advantage (Barrera 1990; Caldwell \& Caldwell 1990; Goodburn et al., 1990). However, in the context of PMTCT, continued utilization of health care services is needed and therefore the exact role of education in reducing LTF needs to be studied further. This is particularly important when PMTCT and ART programs are being rolled out in rural areas among relatively less educated population.

\section{Family's economic status}


HIV infected women staying in families with higher economic status were found to be less likely to be LTF before and after delivery as compared to women belonging to lower economic status. The need for economic empowerment of women to reduce LTF in PMTCT has been recently highlighted from a study in Malawi (Chinkonde et al., 2009). In our study, family's economic status was subjectively assessed by the counselor. This has a limitation when compared with findings from other studies. However, it is important to note that HIV infected women in this study were accessing health care in private sector where they had to bear the cost of obstetric care and medical care for the baby though the services for PMTCT were offered free of cost. During the project implementation it was also noted that the cost of providing obstetric care to HIV infected women was often higher than the cost to HIV negative women in the same private health facility. Our analysis suggests that lack of affordable care in private sector is a significant barrier for continued utilization of PMTCT services by the HIV infected mother and baby. This finding suggests that there is an urgent need for advocacy efforts to reduce the cost of health care for HIV infected women and also underlines the need for public private partnerships so that HIV infected women from different economic backgrounds can access affordable health care.

\section{Woman's stage of pregnancy at the time of registration into the program}

Women who were pregnant for less than 20 weeks at the time of registration into the program were found to be less likely to be LTF compared to women who were pregnant for more than 20 weeks. The cut-off of 20 weeks was selected because medical termination of pregnancy (MTP) in India is legally allowed up-to 20 weeks. It was hypothesized that LTF would be more among women who were registered before 20 
weeks of pregnancy as some of them would opt for terminating their pregnancy and would not return to the PMTCT site. However, the analyses suggest that women who are registered during early pregnancy are more likely to continue utilization of PMTCT services.

Early registration has previously been linked with higher uptake of sdNVP (WHO, 2009). Recognizing the importance of preventing LTF in PMTCT programs, in the recent PMTCT guidelines, WHO recommends to start ARV prophylaxis at 14 weeks despite the lack of direct evidence showing that starting prophylaxis earlier (than 28 weeks) is associated with lower rates of intrauterine transmission (WHO, 2009). Our analysis and program experience suggest that the quality of counselling is important for retaining women in the program. Women in our program who were registered before 28 weeks (and hence were advised to start ARV at 28 week) were less likely to be LTF (data not shown). Women who are registered earlier get the opportunity to have more counseling sessions with the PMTCT counselor and also get more time to cope with their HIV diagnosis which might result into better follow-up. This finding indicates that it is important to concentrate efforts on enrolling women early in the pregnancy in order to retain them in the program.

\section{Partner's HIV status}

Women whose partners were known to be HIV infected were less likely to be LTF before delivery however it was not a significant factor for LTF after delivery. This finding underlines the importance of partner's involvement and support during pregnancy. Partners who are aware of their HIV positive status are known to be more supportive towards their pregnant wives in seeking both antenatal care and care to prevent MTCT 
(Mawar et al., 2007). There are a few studies, mainly from Africa, that documented the benefits of partner involvement within PMTCT set-up. In Zambia and Kenya, for example, women who received counseling along with their partners had improved acceptability of HIV testing, improved uptake of antiretroviral prophylaxis, and better adherence to alternative infant feeding options (Farquhar et al., 2004; Semrau et al., 2005). However there is limited knowledge about the different strategies for partner involvement and testing in the PMTCT set-up. Based on this finding it can be suggested that it is important to counsel partners who are HIV negative or unaware of their HIV status to support and encourage their HIV infected pregnant wives to seek antenatal and postnatal care. Innovative strategies to increase partner involvement in PMTCT are required.

Our study focuses on the demographic risk factors for LTF of HIV infected women before and after delivery in the PMTCT program setting in India. In our analyses we found similar factors associated with LTF both before and after delivery. These factors may be the core factors associated with continued utilization of services for the mother and the child. However, based on program experience, there could be other unmeasured factors such as distance to the clinic, quality of the counseling sessions, non-disclosure to family, and fear of discrimination at hospital that may directly or indirectly affect women's follow-up. Figure 1. shows possible ways in which these factors may be influencing a woman's risk of being LTF from the PMTCT program. One of the possible limitations of this study is that the data were not primarily collected to study LTF in the PMTCT setting. However this is an inherent problem with the research having LTF as the 
study outcome. Therefore it is also essential to take into consideration the unmeasured factors.

Although quality of counseling sessions could not be assessed, there was no significant difference in the LTF across different PMTCT sites suggesting uniformity in the quality of counseling across study sites. In this study we were not able to assess the impact of non-disclosure to family members and experiences of discrimination in the health care setting as risk factors for LTF. Based on the program experience both these factors appear to have direct effect on women's follow-up. Further research is warranted to study the effect of these factors on risk of LTF in the PMTCT context.

Our analyses have identified the need for innovative and effective counseling techniques for less educated women, economic empowerment of women, better strategies to increase up-take of partner's HIV testing and early registration of women in the program for improving utilization of PMTCT services by HIV infected women. The need is even greater for PMTCT programs in public health sector as the women accessing care in the public sector are less likely to be educated and economically more deprived. 
Barrera, A. (1990). The role of maternal schooling and its interaction with public health programs in child health production. Journal of Development Economics 32: 69-91.

Bwirire, L., Fitzgerald, M., et al. (2008). Reasons for loss to follow-up among mothers registered in a prevention-of-mother-to-child transmission program in rural Malawi. Trans R Soc Trop Med Hyg 102(12): 1195-1200.

Caldwell, J. (1979). Education as a factor in mortality decline: An examination of Nigerian data. Population Studies 33: 395-413.

Caldwell, J. (1986). Routes to low mortality in poor countries. Population and Development Review 12: 171-220.

Caldwell, J., \& Caldwell, P. (1988). Women's position and child mortality and morbidity in $L D C^{\prime}$ 's. Paper presented to IUSSP Conference on Women's Position and Demographic Change in the Course of Development, Oslo, Norway.

Caldwell, P., \& Caldwell, J. (1990). Gender implications for survival in South Asia. Health Transition Working Paper No.7, Canberra, Australia.

Chinkonde, J., Sundby, J., et al. (2009). The prevention of mother-to-child HIV transmission programme in Lilongwe, Malawi: why do so many women drop out. Reprod Health Matters 17(33): 143-151.

Cleland, J. (1990). Maternal education and child survival: Further evidence and explanations. In: J. Caldwell, S. Findley, P. Caldwell, G. Santow, W. Cosford, J. Braid, \& D. Broies-Freedman (Eds.), What we know about health transition: The cultural, social, and behavioral determinants of health, Vol. 1 (400-419). Canberra, Australia: The Australian National University Printing Service.

Farquhar, C., Kiarie, J., et al. (2004). Antenatal couple counseling increases uptake of interventions to prevent HIV-1 transmission. J Acquir Immune Defic Syndr 37(5): 16201626.

Goodburn, E., Ebrahim, G., et al. (1990). Strategies educated mothers use to ensure the health of their children. Journal of Tropical Pediatrics 36: 235-239. 
Govindasamy, P. \& Ramesh, B. (1997). Maternal education and the utilization of maternal and child health services in India. (National Family Health Survey Subject Report No.5).

Manzi, M., Zachariah, R., et al. (2005). High acceptability of voluntary counselling and HIV-testing but unacceptable loss to follow up in a prevention of mother-to-child HIV transmission programme in rural Malawi: scaling-up requires a different way of acting. Trop Med Int Health 10(12): 1242-1250.

Mawar, N., Joshi, P., et al. (2007). Concerns and experiences of women participating in a short-term AZT intervention feasibility study for prevention of HIV transmission from mother-to-child. Cult Health Sex 9(2): 199-207.

National AIDS Control Organization. (2007). HIV sentinel surveillance and HIV estimation in India 2007:A technical brief. Retrieved from http://www.nacoonline.org/Quick_Links/Publication/ME_and_Research_Surveillance/Re ports_and_Surveys/HIV_Sentinel_Surveillance_and_HIV_Estimation_2007__A_Technical_Brief/

National AIDS Control Organization. (2008). National AIDS Control Program. Retrieved from http://india.gov.in/sectors/health_family/national_aids.php.

Painter, T., Diaby, K., et al. (2004). Women's reasons for not participating in follow-up visits before starting short course antiretroviral prophylaxis for prevention of mother to child transmission of HIV: qualitative interview study. BMJ 329(543): 1-13.

Perez, F., Mukotekwa, T., et al. (2004). Implementing a rural programme of prevention of mother-to-child transmission of HIV in Zimbabwe: first 18 months of experience. Trop Med Int Health 9(7): 774-783.

Semrau, K., Kuhn, L., et al. (2005). Women in couples antenatal HIV counseling and testing are not more likely to report adverse social events. AIDS 19(6): 603-609.

Sherman, G., Jones, S., et al. (2004). PMTCT from research to reality--results from a routine service. S Afr Med J 94(4): 289-292. 
World Health Organization (2008). Towards universal access: scaling up priority HIV/AIDS interventions in the health sector. Retrieved from http://www.who.int/hiv/pub/2009progressreport/en/index.html

World Health Organization (2009). Rapid Advice: Use of antiretroviral drugs for treating pregnant women and preventing HIV Infection in infants. Retrieved from http://www.who.int/hiv/pub/mtct/advice/en/index.html 


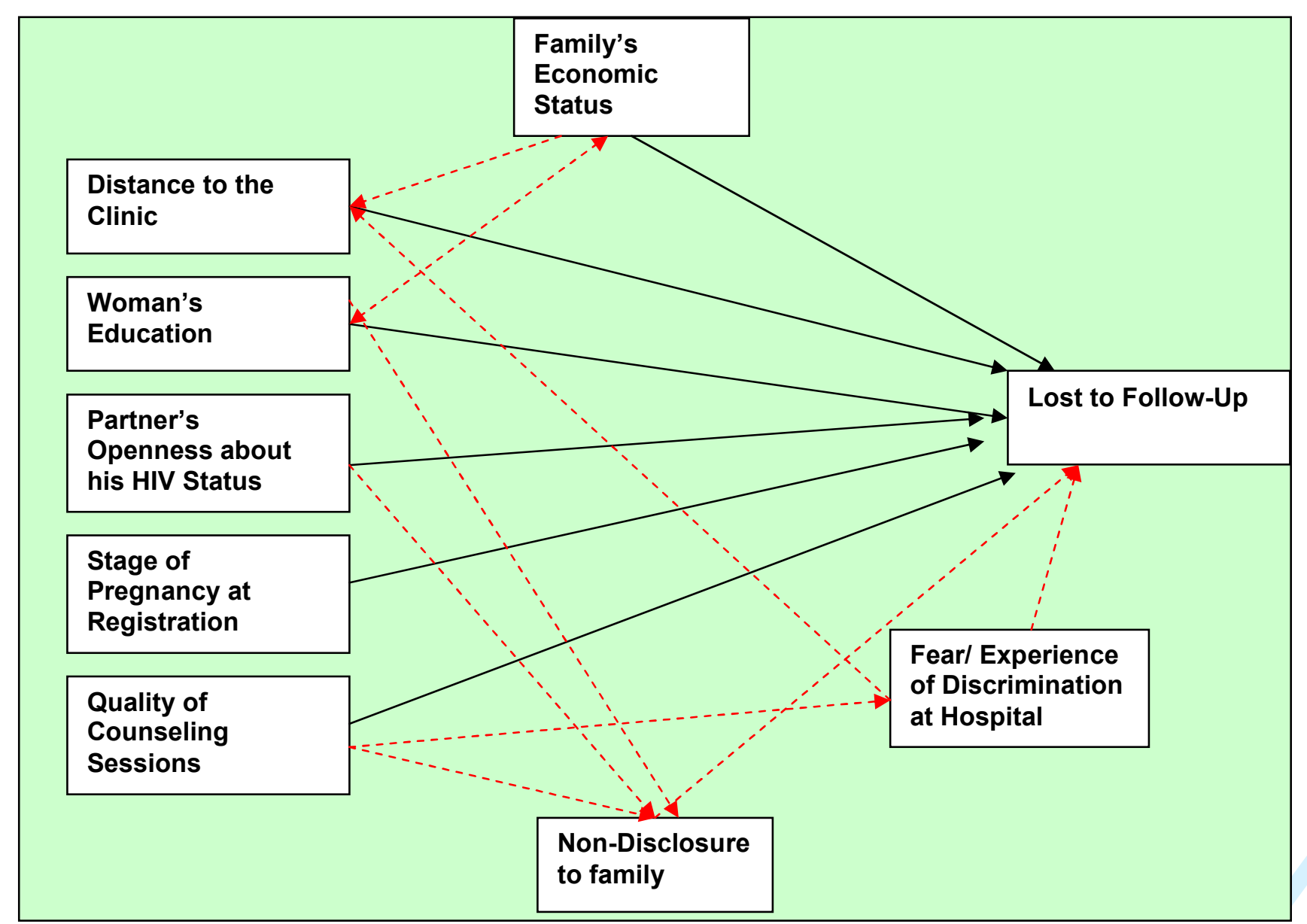

Figure 1: Directed Acyclic Graph showing potential factors that may play role in lost to follow-up of pregnant women registered in the PMTCT program before and after delivery. Solid arrows represent direct effects whereas dashed arrows represent indirect effects. 
Table 1: Crude risk ratios and 95\% confidence intervals of all potential risk factors for being lost to follow-up in HIV positive pregnant women registered at study ANC sites providing PMTCT related care






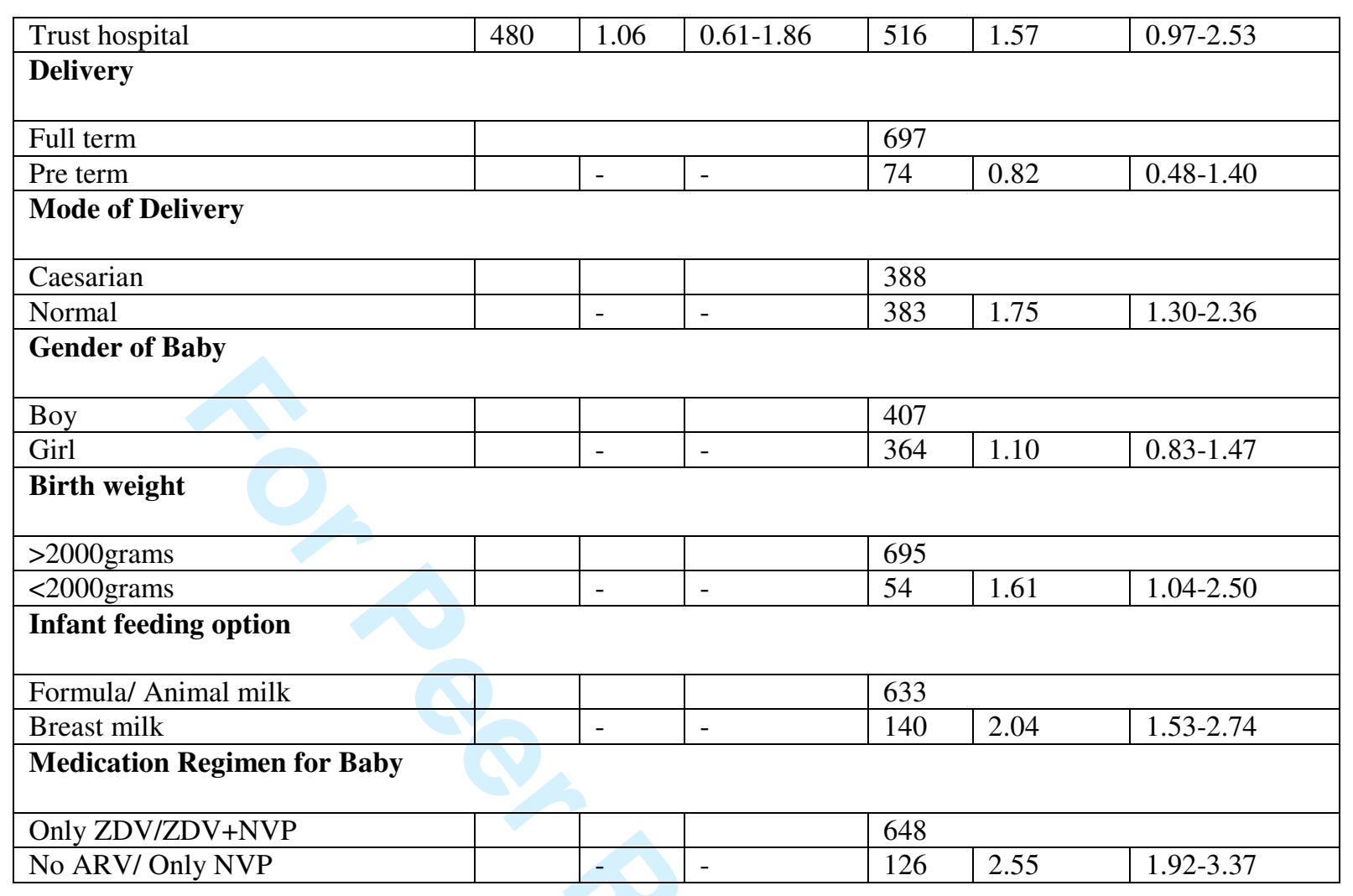


Table 2: Crude and adjusted risk ratios and 95\% confidence intervals for risk factors associated with being lost to follow-up in $734 \mathrm{HIV}$ infected pregnant women registered at study ANC sites providing PMTCT related care (* represents the reference group)

\begin{tabular}{|c|c|c|c|}
\hline & \multirow{2}{*}{$\begin{array}{l}\text { Cases (Number in } \\
\text { Subgroup) }\end{array}$} & \multicolumn{2}{|l|}{ RR (95\% CI) } \\
\hline & & Crude & Adjusted \\
\hline \multicolumn{4}{|l|}{ WomanÕsEducation } \\
\hline Graduate* & $2(117)$ & \multirow{2}{*}{$7.40(1.84-29.68)$} & \multirow{2}{*}{$6.32(1.57-25.44)$} \\
\hline Less than Graduate & $78(617)$ & & \\
\hline \multicolumn{4}{|c|}{ Family's Economic Status } \\
\hline Middle Class/Rich* & $40(537)$ & \multirow{2}{*}{$2.20(1.46-3.76)$} & \multirow{2}{*}{$1.61(1.07-2.42)$} \\
\hline Poor & $35(192)$ & & \\
\hline \multicolumn{4}{|c|}{ Pregnancy Stage at Registration } \\
\hline$<20$ Weeks $*$ & $50(526)$ & \multirow{2}{*}{$1.52(1.00-2.32)$} & \multirow{2}{*}{$2.02(1.34-3.05)$} \\
\hline$>20$ Weeks & $30(208)$ & & \\
\hline \multicolumn{4}{|l|}{ Partner's HIV Status } \\
\hline Positive* & $25(410)$ & \multirow{2}{*}{$2.78(1.78-4.37)$} & \multirow{2}{*}{$2.69(1.71-4.24)$} \\
\hline Negative/Not known & $55(324)$ & & \\
\hline
\end{tabular}


Table 3: Crude and adjusted risk ratios and 95\% confidence intervals for risk factors associated with being lost to follow-up after delivery in 769 HIV infected women registered at study ANC sites providing PMTCT related care (* represents the reference group)

\begin{tabular}{|c|c|c|c|}
\hline & \multirow{2}{*}{$\begin{array}{l}\text { Cases (Number in } \\
\text { Subgroup) }\end{array}$} & \multicolumn{2}{|l|}{ RR $(95 \%$ CI) } \\
\hline & & Crude & Adjusted \\
\hline \multicolumn{4}{|l|}{ Woman's Education } \\
\hline Graduate* & $12(122)$ & \multirow{2}{*}{$2.17(1.24-3.79)$} & \multirow{2}{*}{$1.82(1.03-3.22)$} \\
\hline Less than Graduate & $138(647)$ & & \\
\hline \multicolumn{4}{|c|}{ Family's Economic Status } \\
\hline Middle Class/Rich* & $92(560)$ & \multirow{2}{*}{$1.65(1.23-2.20)$} & \multirow{2}{*}{$1.42(1.05-1.91)$} \\
\hline Poor & $56(207)$ & & \\
\hline \multicolumn{4}{|c|}{ Pregnancy Stage at Registration } \\
\hline$<20$ weeks* & $20(179)$ & \multirow{2}{*}{$1.98(1.27-3.07)$} & \multirow{2}{*}{$1.75(1.12-2.73)$} \\
\hline$>20$ weeks & $130(588)$ & & \\
\hline
\end{tabular}

